\title{
Genetic variations in genes involved in heparan sulphate biosynthesis are associated with Plasmodium falciparum parasitaemia: a familial study in Burkina Faso
}

\author{
Alexandre Atkinson ${ }^{1,3}$, Séverine Garnier ${ }^{1,3}$, Sarwat Afridi ${ }^{1,3}$, Francis Fumoux ${ }^{2,3}$ and Pascal Rihet ${ }^{1,3^{*}}$
}

\begin{abstract}
Background: There is accumulating evidence that host heparan sulphate proteoglycans play an important role in the life cycle of Plasmodium through their heparan sulphate chains, suggesting that genetic variations in genes involved in heparan sulphate biosynthesis may influence parasitaemia. Interestingly, Hs3st3al and Hs3st3b1 encoding enzymes involved in the biosynthesis of heparan sulphate are located within a chromosomal region linked to Plasmodium chabaudi parasitaemia in mice. This suggests that HS3ST3A1 and HS3ST3B1 may influence P. falciparum parasitaemia in humans.
\end{abstract}

Methods: Polymorphisms within HS3ST3A1 and HS3ST3B1 were identified in 270 individuals belonging to 44 pedigrees and living in Burkina Faso. Linkage and association between parasitaemia and the polymorphisms were assessed with MERLIN and FBAT. A genetic interaction analysis was also conducted based on the PGMDR approach.

Results: Linkage between $P$. falciparum parasitaemia and the chromosomal region containing HS3ST3A1 and HS3ST3B1 was detected on the basis of the 20 SNPs identified. In addition, rs28470223 located within the promoter of HS3ST3A1 was associated with P. falciparum parasitaemia, whereas the PGMDR analysis revealed a genetic interaction between HS3ST3A1 and HS3ST3B1. Seventy-three significant multi-locus models were identified after correcting for multiple tests; 37 significant multi-locus models included rs28470223, whereas 38 multi-locus models contained at least one mis-sense mutation within HS3ST3B1.

Conclusion: Genetic variants of HS3ST3A1 and HS3ST3B1 are associated with P. falciparum parasitaemia. This suggests that those variants alter both the function of heparan sulphate proteoglycans and $P$. falciparum parasitaemia.

Keywords: HS3ST3A1, HS3ST3B1, Heparan sulphate biosynthesis, Plasmodium falciparum, Malaria, Parasitaemia, Family-based association, Genetic interaction

\section{Background}

Plasmodium falciparum malaria is transmitted to humans through the bite of infected female Anopheles mosquitoes. Sporozoites that have been injected into the skin migrate from the site of injection, and reach the liver, where they invade hepatocytes and change into merozoites; merozoites penetrate and replicate inside

\footnotetext{
* Correspondence: pascal.rihet@univmed.fr

'UMR928-TAGC, INSERM, 163 Av de Luminy, Marseille F-13288, France Full list of author information is available at the end of the article
}

red blood cells. Heparan sulphate proteoglycans (HSPGs) may play an important role in the biology of Plasmodium through their carbohydrate chains (heparan sulphate) in both the mammalian host and the vector. Anopheles heparan sulphate has been shown to bind circumsporozoite protein (CSP), suggesting a role for the carbohydrate chains within Anopheles salivary glands for infection and transmission of the parasite [1]. CSP also interacts with the HSPGs on host liver cells, and this interaction has been shown to determine the choice 
between migrating through or invading the cell [2]. Highly sulphated HSPGs of hepatocytes activates the rodent malaria parasite Plasmodium berghei for invasion, whereas the parasite migrates through cells with low sulphated HSPGs in skin and endothelium [2]. In addition, a $P$. falciparum merozoite antigen (EBA-140) has been shown to bind to red blood cells in a heparan sulphate-manner, whereas soluble heparan sulphate and heparin inhibit the merozoite invasion into red blood cells $[3,4]$. Finally, heparan sulphate is thought to be a receptor for PfEMP1 expressed on infected red blood cells (iRBC), and to mediate the binding of iRBC on endothelial cells or other red blood cells [4-6].

These observations suggest that the outcome of malaria infection may be influenced by variations in the biosynthesis of heparan sulphate, owing to genetic variations within genes encoding the enzymes involved. These include $\mathrm{O}$-sulphotransferases, which catalyze 2-O, 6-O, or 3-O sulphation [7]. The O-sulphation steps are the last steps of the synthesis of heparan sulphate (HS); the sulphation level is a measure of the completion of this synthesis, and is thought to influence the binding properties and therefore the function of HSPGs [7]. The 3-O-sulphation is thought to be a rare event, whereas the 3-O sulphated HS has been shown to serve as an entry receptor of Herpes Simplex Virus 1 (HSV-1) [8]. Interestingly, whereas only one 2-O sulphotransferase, and one 6-O sulphotransferase are known, seven isoforms of 3-O sulphotransferases have been reported $[7,9]$. The genes encoding the 3-O sulphotransferases are located in different chromosomal regions except for HS 3ST $3 A 1$ and HSSST3B1, and the only significant sequence homology between these proteins occurs in the sulphotransferase domains. Nevertheless, HS3ST3A1 and HS3ST3B1, which encode the 3-O sulphotransferases 3-OST-3A1 and 3-OST-3B1 respectively, are 700 $\mathrm{kb}$ apart in the same chromosomal region, and show a high sequence identity [10]. 3-OST-3B1, which has a sulphotransferase domain $99.2 \%$ identical to that of 3 OST-3A1, sulphates an identical disaccharide [11]. Recently, HS3ST3A1 that encodes 3-OST-3A1 has been associated with mother-to-child transmission of human immunodeficiency virus (HIV) through a genome-wide association study [12], whereas HSPGs promotes HIV penetration through endothelial cells in a heparan sulphate-manner [13]. This supports the hypothesis that genetic variations within the genes encoding 3-O-sulphotransferases may affect the susceptibility to infectious diseases, such as malaria.

There is a growing body of evidence for human genetic factors controlling the outcome of infection. Familial aggregation and segregation analyses showed the existence of a genetic component of phenotypes related to $P$. falciparum malaria resistance or susceptibility $[14,15]$. Several candidate genes have been associated with resistance against severe malaria [16]. Linkage or association analyses mapped various loci controlling mild malaria and/or parasitaemia in humans [15,17-19]. The first chromosomal regions that showed linkage to mild malaria and/or parasitaemia were 6p21.3 and 5q31-q33 [15,17,20,21]. However, a limited number of confirmed alleles involved in human malaria have been identified [22,23]. In crosses between genetically defined strains of mice, chromosomal regions responsible for the genetic variance of complex traits can be mapped as quantitative trait loci (QTL) in experimental populations available for precise study under defined conditions. Linkage analyses based on experimental crosses have been done in mice, leading to the mapping of loci controlling Plasmodium chabaudi parasitaemia (Char1-10) or cerebral malaria [22,24,25]. Notably, such analyses mapped two loci on chromosomes 17 (Char3) and 11 (Char8), which show extensive conservation of synteny with human chromosomes $6 \mathrm{p} 21.3$ and 5q31-q33, respectively [26,27]. The $95 \%$ confidence interval of Char8 (chromosome 11 between D11Mit231 and D11Mit30) contained mostly genes, the orthologs of which are located in human chromosome 5q31-q33. Nevertheless, it also contained genes, the orthologs of which are located in human chromosome 17; these include Hs3st3a 1 and Hs3st3b1, which encode 3-O sulphotransferases in mice $[26,27]$, suggesting that genetic variations within $H S 3 S T 3 A 1$ and $H S 3 S T 3 B 1$ may influence parasitaemia in humans. This hypothesis was further supported by a linkage study based on microsatellite markers in humans (P.Rihet, unpublished data). This prompted us to screen HS3ST3A1 and HSSST3B1 in a population living in an endemic area in Burkina Faso to identify polymorphisms, to evaluate their linkage and association with parasitaemia, and gene-gene interactive effects by using the pedigreebased generalized multifactor dimensionality reduction (PGMDR) method [28].

\section{Methods}

\section{Subjects}

The study subjects live in a rural area, Logoforousso, a village to the south-west of Bobo-Dioulasso (Burkina Faso). The population and the area of parasite exposure have been extensively described [29]. Volunteer families were randomly selected from 3,500 inhabitants. Informed consent was then obtained individually from all participants or their parents. The protocol was approved by the national medical authorities of Burkina Faso. Four mosquito capture sites were chosen, and mosquitoes were collected outdoors 4 days each month (during two nights every 2 weeks). The inoculation rate 
was 230 infective bites per person per year. The study population comprised 270 subjects from 44 pedigrees corresponding to 71 nuclear families. Eighty-seven parents and 183 siblings were available for genotyping and retained for linkage analysis. The mean age of the siblings was $9.9 \pm 4.4$ (three to 25 years).

\section{Phenotyping}

Parasitaemia was measured as described [29]. During two years, each family in the rural area was visited 28 times. Blood samples were taken from all individuals present, and only asymptomatic $P$. falciparum parasitaemia measurements were considered in this study. The mean number of asymptomatic $P$. falciparum parasitaemia measurements per subject was $14.9+8.1$ (range one to 28). The parasitaemia was defined as the number of parasitized erythrocytes observed per microlitre in thin blood films. The analysis was conducted on a logarithmic transformation of parasitaemia adjusted for seasonal transmission and for age that showed a significant effect on parasitaemia $\left(\mathrm{P}<10^{-4}\right)$. The standardized residual was the phenotype used for linkage and association analyses.

\section{Genotyping}

Each subject underwent a venipuncture, and DNA was extracted from mononuclear cells separated by FicollHypaque density gradient as described [15]. DNA concentration was assessed with a biophotometer (Eppendorf, Le Pecq, France). The DNA samples $(\mathrm{n}=270)$ were subjected to prior whole-genome amplification with the Illustra GenomiPhi V2 DNA Amplification Kit from General Electric Healthcare (GE Healthcare Biosciences, Pittsburgh, PA, USA) before genotyping. To identify mutations in the promoter region, in all the coding regions and the intron-exon border, defined PCR products were sequenced. All primer pairs were designed with the PRIMER 3 program [30]. There is a strong homology between HS3ST3A1 coding sequence and that of $H S 3 S T 3 B 1$, particularly between exon 2 sequences [10]. Therefore, a first set of primers corresponding to non-coding regions that display sequence variation were designed. PCR was performed with a first set of primers leading to a large product, whereas sequencing reaction was performed with additional internal primers (Additional file 1). Before starting the sequencing reaction, the PCR products were purified with the Qiagen QIAquick PCR purification kit (Qiagen, Hilden, Germany) and quantified by $2 \%$ agarose gel electrophoresis. Sequencing reaction was performed with the CEQ DTCS kit (Beckman Coulter, Fullerton, CA, USA) and a CEQ 8800 automated fluorescent sequencer (Beckman Coulter).

\section{Allele frequencies, haplotype reconstruction and linkage disequilibrium analysis}

The compatibility with Mendelian inheritance of marker alleles was checked with the FBAT and MERLIN programs [31,32]. All genotypes passed a Mendelian check with the program FBAT. Using MERLIN, improbable recombination events from SNP maps were checked to detect genotyping errors.

Allele frequencies were calculated by gene counting and deviation from Hardy-Weinberg equilibrium was tested using a Chi-2-test with 1 degree of freedom. Haplotypes were generated based on family genotypic data with MERLIN. Pair-wise LD (Linkage Disequilibrium) was calculated with Haploview and graphical overview of linkage disequilibrium (GOLD) [33,34]. LD between pairs of biallelic markers was tested by the $\mathrm{r} 2$ statistic, $\mathrm{r}^{2}$ $=(\mathrm{p} 11 \mathrm{p} 22-\mathrm{p} 12 \mathrm{p} 21)^{2} / \mathrm{p} 1 \mathrm{p} 2 \mathrm{q} 1 \mathrm{q} 2$, where $\mathrm{p} 11, \mathrm{p} 22, \mathrm{p} 12$, and $\mathrm{p} 21$ were two-locus haplotype frequencies, and p1, $\mathrm{p} 2$, $\mathrm{q} 1$, and $\mathrm{q} 2$ were allele frequencies. $\mathrm{r}^{2}$ is the standard $\mathrm{c}^{2}$ statistic divided by the number of chromosomes in the sample. It ranges from 0 to 1 . When $r^{2}$ is 1 , SNPs are in complete LD.

\section{Statistical analyses}

Multipoint linkage analyses were performed for sibship and half-sibship data using the software package the MERLIN Package [31]. The regression-based procedure was used for quantitative trait linkage analysis [35]. Combined association and linkage analyses of quantitative traits were carried out using the FBAT program and the QTDT program $[32,36]$. FBAT calculates a Z score and a two-side $P$ value based on a normal approximation. Association in the presence of linkage was assessed using the orthogonal model released in the QTDT program [36]. $P$ values were calculated using the likelihood-ratio criterion.

Genetic interactions were analysed by using the pedigree-based generalized multifactor dimensionality reduction method (PGMDR) [28]. The PGMDR is a scorebased MDR method that uses the same data reduction strategy as does the original MDR method to detect non-linear genetic interactions [37]. Briefly, the informative siblings were randomly divided into nearly 10 nearly equal subsets, and the cross-validation was repeated 10 times. Each time, nine subsets were used as the training set to construct a binary model with high risk and low risk genotype combinations, while the last subset was considered the testing set. The odds ratio, the corresponding $95 \%$ confidence interval, and the $P$ value were calculated for the training set. The testing set was used to estimate the prediction accuracy of the model, which is the ratio of correct classifications to the total number of instances classified. The non-parametric sign test was 
used to evaluate the significance of the prediction accuracy.

The false discovery rate (FDR) procedure was performed to account for the multiple tests performed [38]; an FDR of 5\% and an FDR of 10\% were applied. Multiple test corrections were carried out for all the linkage, association, and interaction analyses.

\section{Results}

\section{Descriptive analyses}

Twenty polymorphisms were identified in the promoter, the exons, the intron-exon junctions, and the 3 ' untranslated region of HSSST3A1 and HS3ST3B1 (Table 1). Notably, some SNPs annotated both in HS3ST3A1 and HS3ST3B1 were found in either HS3ST3A1 or HS3ST3B1 (Figure 1). All SNPs passed a Mendelian check, and the detectable genotype errors were $<0.1 \%$ based on improbable recombination events from dense SNP maps. There was no deviation from the HardyWeinberg equilibrium. Figure 2 shows pair-wise linkage disequilibrium coefficients. There was a significant linkage disequilibrium between some SNPs within either HS3ST3A1 or HS3ST3B1, whereas no linkage disequilibrium was detected between HS3ST3A1 and HS3ST3B1. In particular, there was a highly significant linkage disequilibrium between rs3744337 and rs3744335 $\left(\mathrm{r}^{2}=0.72\right.$; $P<0.0001$ ) within HS3ST3A1; there was also a highly significant linkage disequilibrium between rs2072243 and $\mathrm{rs} 2072242\left(\mathrm{r}^{2}=0.64 ; \mathrm{P}<0.0001\right)$ within HS3ST3B1.

\section{Linkage and association analyses}

A regression-based procedure was applied for multipoint linkage analysis using MERLIN. Based on the analysis of the 20 SNPs, parasitaemia was genetically linked to the region containing HSSST3A1 and HS3ST3B1 after applying an FDR of $10 \%$ (Table 2), with a peak of linkage at rs61729712 (LOD score $=1.207, P=0.009$ ). A linkage was also detected based on the analysis of the 10 SNPs with the highest Minor Allele Frequency (MAF) after applying a FDR of $5 \%$ (Table 3); the peak of linkage was, nevertheless, at rs115229628 $($ LOD score $=1.409, P$ $=0.005$ ).

Combined linkage and association between each SNP and parasitaemia was further evaluated. FBAT showed evidence of linkage and association between rs28470223 and parasitaemia $(P=0.005)$, whereas the other SNPs were not associated with parasitaemia $(P>0.09)$. The allele $\mathrm{C}$ was negatively associated with parasitaemia $(\mathrm{Z}=$ -2.77; $P=0.005$ ), whereas the allele $\mathrm{T}$ was positively associated with parasitaemia $(\mathrm{Z}=2.77 ; P=0.005)$. This result remained significant after applying an FDR of $5 \%$, and confirmed the linkage signal detected with MERLIN. Furthermore, the linkage was taken into account to test the association between each SNP and parasitaemia by using QTDT; an association in the presence of linkage was detected for rs28470223 $(P<0.003)$ after applying an FDR of $5 \%$.

\section{Interaction analysis}

The epistatic effect of HS3ST3A1 and HS3ST3B1 SNPs was investigated, based on the knowledge of the biological activity of HSSST3A1 and HS3ST3B1. Two-, three-, four-, and five-locus models were evaluated. Additional file 2 shows the best models identified on the basis of the classification parameters with the training set (Odds Ratio and $P$ value) and the cross-validation analysis with the testing set (prediction accuracy and sign test $P$ value) after applying a FDR of 5\%. The analysis revealed 73 significant multi-locus models, which could be consistently cross-validated (Additional file 2). In other words, the analysis of the training set yielded 73 binary models with high and low risk genotype combinations, which were significantly associated with parasitaemia, and which were validated based on the testing set. All the significant multi-locus models included SNPs located in both HS3ST3A1 and HS3ST3B1. The significant models included 19 of the 20 SNPs. These include: i) rs3744337, rs3744335, rs28470223, rs78863672, rs2072243, rs2072242, and rs115229628 that are located within the 5'UTR region of either HS3ST3A1 or HS3ST3B1: ii) rs3785655 and rs7379332 that are within the 3'UTR region of HS3ST3B1; iii) rs9906855, rs62057033, rs61732181, rs617444056, rs62636622 and rs55688668 that are synonymous mutations of either HS3ST3A1 or HS3ST3B1; and iv) rs62636623, rs62056073, rs61729712, and rs9906590 that are missense mutations within HS3ST3B1. Interestingly, rs28470223 was in 37 of the 73 significant multi-locus models, and 38 multi-locus models contained at least one mis-sense mutation.

\section{Discussion}

This is apparently the first study to investigate the association between a phenotype related to malaria susceptibility and genes involved in HS biosynthesis. Two genes $700 \mathrm{~kb}$ apart, which encode 3-O sulphotransferases involved in the synthesis of HS (HS3ST3A1 and HS3ST3B1), were considered as candidate genes.

First, $P$. falciparum parasitaemia was found to be genetically linked to HS3ST3A1 and HS3ST3B1 polymorphisms based on a multipoint linkage analysis. This result is consistent with linkage studies based on microsatellite markers in mice [26,27] and humans (P. Rihet, unpublished data). This suggests that polymorphisms within the chromosomal region may partly explain the variance of parasitaemia, and may affect resistance against malaria. In the same way, rs6503319, which is located in the human chromosomal region genetically 
Table 1 Overview of the polymorphisms genotyped in the African population

\begin{tabular}{|c|c|c|c|c|c|c|}
\hline SNP Id ${ }^{a}$ & SNP rs\# & GRCh37 location (bp) & Localization within gene & Alleles $^{\text {b }}$ & $\mathrm{MAF}^{\mathrm{c}}$ & Molecular change \\
\hline & rs1047933 & 13399333 & HS3ST3A1 3'UTR & $\mathrm{T}>\mathrm{A}$ & - & - \\
\hline & rs67951062 & 13399487 & HS3ST3A1 3'UTR & $->\pi T$ & - & - \\
\hline & rs11385090 & 13399495 & HS3ST3A1 3'UTR & $A>-$ & - & - \\
\hline & rs67556828 & 13399497 & HS3ST3A1 3'UTR & $->T$ & - & - \\
\hline & rs56160453 & 13399536 & HS3ST3A1 ex2 & $A>G$ & - & mis-sense $\mathrm{H}$ [His] $\Rightarrow \mathrm{R}[\mathrm{Arg}]$ \\
\hline & rs56184152 & 13399569 & HS3ST3A1 ex2 & $C>T$ & - & mis-sense $P[$ Pro $] \Rightarrow L[$ Leu $]$ \\
\hline \multirow[t]{4}{*}{1} & rs62057033 & 13399616 & HS3ST3A1 ex2 & $\mathrm{T}>\mathrm{A}$ & 0.069 & Synonymous \\
\hline & rs55688668 & 13399778 & HS3ST3A1 ex2 & $G>A$ & - & Synonymous \\
\hline & rs56408399 & 13399795 & HS3ST3A1 ex2 & $C>T$ & - & mis-sense $R[A r g] \Rightarrow W[$ Trp] \\
\hline & rs61729712 & 13399884 & HS3ST3A1 ex2 & $G>A$ & - & mis-sense $\mathrm{S}[\mathrm{Ser}] \Rightarrow \mathrm{N}[\mathrm{Asn}]$ \\
\hline \multirow[t]{3}{*}{2} & rs61732181 & 13399928 & HS3ST3A1 ex2 & $G>A$ & 0.043 & Synonymous \\
\hline & rs55888783 & 13400004 & HS3ST3A1 ex2 & $A>G$ & - & mis-sense $D[$ Asp $] \Rightarrow G[G l y]$ \\
\hline & rs56307410 & 13400014 & HS3ST3A1 ex2 & $G>A$ & - & missense $V[\mathrm{Val}] \Rightarrow \mathrm{M}[\mathrm{Met}]$ \\
\hline 3 & rs61744056 & 13400057 & HS3ST3A1 ex2 & $C>T$ & 0.005 & Synonymous \\
\hline \multirow[t]{4}{*}{4} & rs8080565 & 13400153 & HS3ST3A1 intron & $C>T$ & 0.106 & - \\
\hline & rs60532842 & 13504194 & HS3ST3A1 ex1 & $C>A$ & - & mis-sense A $[\mathrm{Ala}] \Rightarrow \mathrm{S}[\mathrm{Ser}]$ \\
\hline & rs 28663356 & 13504408 & HS3ST3A1 ex1 & $C>A$ & - & Synonymous \\
\hline & rs73298111 & 13504474 & HS3ST3A1 5'UTR & $C>T$ & - & - \\
\hline \multirow[t]{3}{*}{5} & rs3744337 & 13504665 & HS3ST3A1 5'UTR & $C>T$ & 0.330 & - \\
\hline & rs3744336 & 13504689 & HS3ST3A1 5'UTR & $A>T$ & - & - \\
\hline & rs34011501 & 13504833 & HS3ST3A1 5'UTR & $->T$ & - & - \\
\hline 6 & rs3744335 & 13504884 & HS3ST3A1 5'UTR & $A>C$ & 0.320 & - \\
\hline 7 & rs 28470223 & 13505023 & HS3ST3A1 5'UTR & $C>T$ & 0.161 & - \\
\hline \multirow[t]{3}{*}{8} & rs78863672 & 13505237 & HS3ST3A1 5'UTR & $G>T$ & 0.433 & - \\
\hline & rs67848311 & 13505244 & intergenic & $C>-$ & - & - \\
\hline & rs58718148 & 13505245 & intergenic & $C>-$ & - & - \\
\hline 9 & rs2072243 & 14204380 & HS3ST3B1 5'UTR & $C>T$ & 0.150 & - \\
\hline 10 & rs2072242 & 14204410 & HS3ST3B1 5'UTR & $\mathrm{T}>\mathrm{C}$ & 0.184 & - \\
\hline \multirow[t]{2}{*}{11} & rs115229628 & 14204423 & HS3ST3B1 5'UTR & $G>A$ & 0.097 & - \\
\hline & rs72241295 & $14204547-14205186$ & HS3ST3B1 5'UTR $\rightarrow$ ex1 & 639 bp del & - & Large deletion \\
\hline 12 & rs62636623 & 14205082 & HS3ST3B1 ex1 & $\mathrm{G}>\mathrm{C}$ & 0.020 & mis-sense $G$ [Gly] $\Rightarrow R$ [Arg] \\
\hline 13 & rs62636622 & 14205168 & HS3ST3B1 ex1 & $G>A$ & 0.021 & Synonymous \\
\hline 14 & rs62056073 & 14248376 & HS3ST3B1 ex2 & $A>G$ & 0.009 & mis-sense I [lle] $\Rightarrow \vee$ [Val] \\
\hline \multirow[t]{4}{*}{15} & rs9906855 & 14248423 & HS3ST3B1 ex2 & $C>T$ & 0.173 & synonymous \\
\hline & rs56307410 & 14248466 & HS3ST3B1 ex2 & $G>A$ & - & mis-sense $V[\mathrm{Val}] \Rightarrow \mathrm{M}[\mathrm{Met}]$ \\
\hline & rs55888783 & 14248476 & HS3ST3B1 ex2 & $A>G$ & - & mis-sense $D[$ Asp] $\Rightarrow G$ [Gly] \\
\hline & rs61732181 & 14248552 & HS3ST3B1 ex2 & $\mathrm{G}>\mathrm{T} / \mathrm{C}$ & - & synonymous \\
\hline \multirow[t]{2}{*}{16} & rs61729712 & 14248596 & HS3ST3B1 ex2 & $G>A$ & 0.006 & mis-sense $S[$ Ser $] \Rightarrow N[$ Asn $]$ \\
\hline & rs56408399 & 14248685 & HS3ST3B1 ex2 & $C>T$ & - & mis-sense $R[$ Arg] $\Rightarrow W[$ Trp] \\
\hline \multirow[t]{2}{*}{17} & rs55688668 & 14248702 & HS3ST3B1 ex2 & $G>A$ & 0.066 & synonymous \\
\hline & rs61741326 & 14248864 & HS3ST3B1 ex2 & $\mathrm{T}>\mathrm{A}$ & - & synonymous \\
\hline \multirow[t]{3}{*}{18} & rs9906590 & 14248877 & HS3ST3B1 ex2 & $G>A$ & 0.098 & mis-sense $\mathrm{E}[\mathrm{Glu}] \Rightarrow \mathrm{K}[$ Lys $]$ \\
\hline & rs56184152 & 14248911 & HS3ST3B1 ex2 & $C>T$ & - & mis-sense $P[$ Pro $] \Rightarrow L[$ Leu $]$ \\
\hline & rs56160453 & 14248944 & HS3ST3B1 ex2 & $A>G$ & - & missense $\mathrm{H}[\mathrm{His}] \Rightarrow \mathrm{R}[\mathrm{Arg}]$ \\
\hline 19 & rs3785655 & 14249167 & HS3ST3B1 3'UTR & $C>T$ & 0.059 & - \\
\hline 20 & rs7379332 & 14249433 & HS3ST3B1 3'UTR & $C>T$ & 0.070 & - \\
\hline
\end{tabular}

a Detected SNPs are shown. ${ }^{\mathrm{b}}$ Major allele $>$ minor allele. ${ }^{\mathrm{c}}$ Allele frequency that was calculated in the study population is shown

linked to parasitaemia (P.Rihet, unpublished data), has been associated with severe malaria [39]. It should be stressed, however, that the location of the peak of linkage depends on the SNPs included in the analysis. This indicates either that the linkage analysis does not accurately locate the causal polymorphisms, or that 


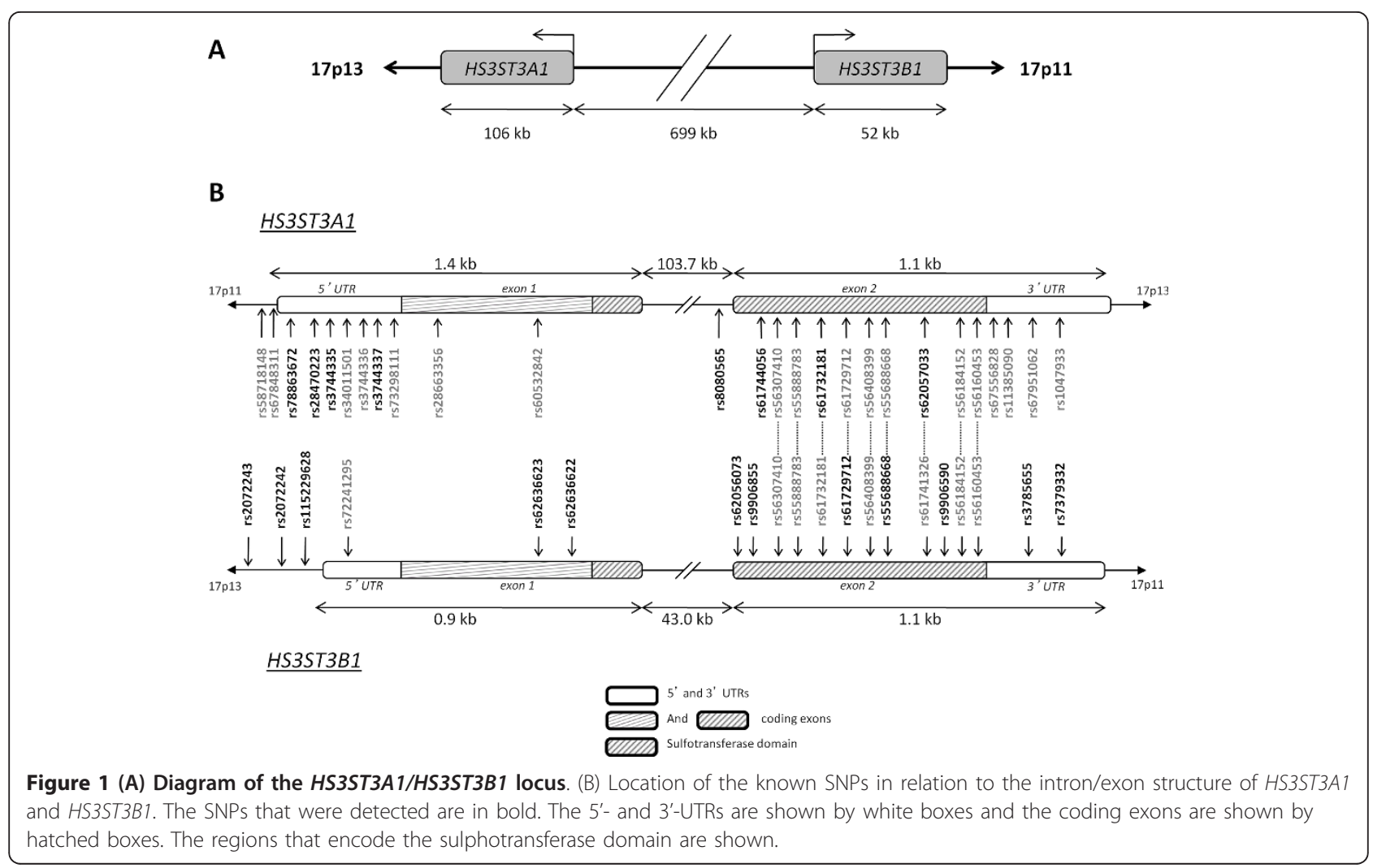

several polymorphisms within the region may influence parasitaemia.

Second, linkage and association between parasitaemia and rs28470223 was detected. Thus, this one-locus analysis confirmed the linkage signal obtained with the multipoint analysis. Furthermore, rs 28470223 , which is located within the promoter of HS3ST3A1, was associated in the presence of linkage with parasitaemia. This supports the hypothesis that rs28470223 alters both the expression of HS3ST3A1 and parasitaemia. Functional studies will be required to evaluate whether rs 28470223 affects the binding of a transcription factor, and the

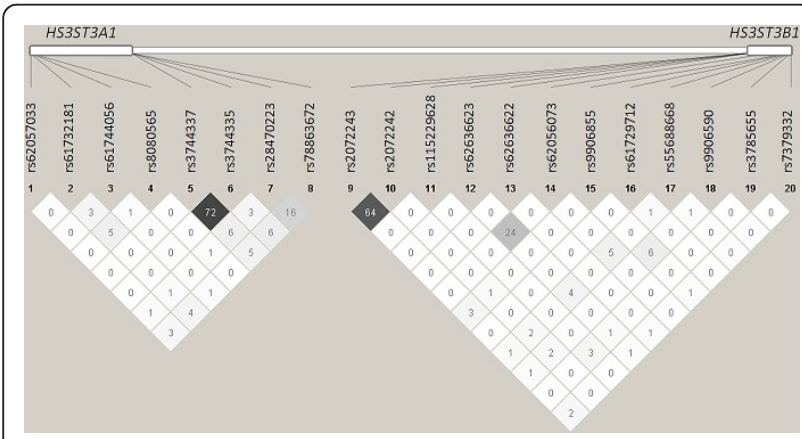

Figure 2 Pair-wise linkage disequilibrium map within HS $3 S T 3 A 1$ and HS3ST3B1. Dark denotes highly significant linkage

disequilibrium. $R^{2}$ multiplied by 100 value is shown.
Table 2 Multi-point linkage analyses of parasitaemia to HS3ST3A1 and HS3ST3B1 based on the 20 SNPS

\begin{tabular}{|c|c|c|c|}
\hline SNP Id & SNP rs\# & LOD & $P$ value \\
\hline 1 & rs62057033 & 0.578 & 0.05 \\
\hline 2 & rs61732181 & 0.578 & 0.05 \\
\hline 3 & rs61744056 & 0.610 & 0.05 \\
\hline 4 & rs8080565 & 0.578 & 0.05 \\
\hline 5 & rs3744337 & 0.682 & 0.04 \\
\hline 6 & rs3744335 & 0.634 & 0.04 \\
\hline 7 & rs28470223 & 0.636 & 0.04 \\
\hline 8 & rs78863672 & 0.631 & 0.04 \\
\hline 9 & rs2072243 & 1.111 & $0.012^{a}$ \\
\hline 10 & rs2072242 & 1.106 & $0.012^{a}$ \\
\hline 11 & rs115229628 & 1.144 & $0.011^{a}$ \\
\hline 12 & rs62636623 & 1.138 & $0.011^{a}$ \\
\hline 13 & rs62636622 & 1.140 & $0.011^{a}$ \\
\hline 14 & rs62056073 & 1.136 & $0.011^{\mathrm{a}}$ \\
\hline 15 & rs9906855 & 1.157 & $0.010^{a}$ \\
\hline 16 & rs61729712 & 1.207 & $0.009^{a}$ \\
\hline 17 & rs556888668 & 1.199 & $0.009^{a}$ \\
\hline 18 & rs9906590 & 1.199 & $0.009^{a}$ \\
\hline 19 & rs3785655 & 1.200 & $0.009^{a}$ \\
\hline 20 & rs7379332 & 1.201 & $0.009^{a}$ \\
\hline
\end{tabular}

${ }^{\mathrm{a}}$ Significant $P$ value after applying an FDR of $10 \%$ 


\begin{tabular}{|c|c|c|c|}
\hline SNP Id & SNP rs\# & LOD & $P$ value \\
\hline 4 & rs8080565 & 0.548 & 0.06 \\
\hline 5 & rs3744337 & 0.735 & 0.03 \\
\hline 6 & rs3744335 & 0.684 & 0.04 \\
\hline 7 & rs28470223 & 0.749 & 0.03 \\
\hline 8 & rs78863672 & 0.743 & 0.03 \\
\hline 9 & rs2072243 & 1.258 & $0.008^{a}$ \\
\hline 10 & rs2072242 & 1.361 & $0.006^{a, b}$ \\
\hline 11 & rs115229628 & 1.409 & $0.005^{a, b}$ \\
\hline 15 & rs9906855 & 0.684 & 0.04 \\
\hline 18 & rs9906590 & 0.684 & 0.04 \\
\hline
\end{tabular}

${ }^{\text {a }}$ Significant $P$ value after applying an FDR of $10 \%$

${ }^{\text {b }}$ Significant $P$ value after applying an FDR of $5 \%$

level of gene expression. However, it cannot be excluded that rs28470223 is in linkage disequilibrium with the causal polymorphism.

Third, given that HS3ST3A1 and HS3ST3B1 encode enzymes with a nearly identical activity, a gene-by-gene interaction analysis was conducted based on the PGMDR approach [28]. Two-, three-, four, and fivelocus interactions were systematically evaluated. Seventy-three significant multi-locus models, which included SNPs found in both HS3ST3A1 and HS3ST3B1, were identified. This supports the hypothesis of epistatic interaction between HS3ST3A1 and HS3ST3B1. In addition, 37 out of the 73 significant multi-locus models included rs28470223 located in the promoter of HS3ST3A1, further supporting the hypothesis of a particular role for rs28470223. Other SNPs located in the promoter of either HS3ST3A1 or HS3ST3B1 and synonymous mutations, which may alter gene expression levels, were also included in several significant multi-locus models. Moreover, 38 multi-locus models contained at least one mis-sense mutation. This suggests a possible functional role of rs62636623, rs62056073, rs61729712, and rs9906590, which alter the sequence of amino acids. rs62056073, rs61729712, and rs9906590 are of major interest because they affect the sulphotransferase domain, suggesting that they may alter the enzymatic activity. Interestingly, site-directed mutagenesis experiments have demonstrated that several amino acid changes in the sulphotransferase domain dramatically reduce the enzymatic activity [40]. In addition, rs62056073, rs61729712, and rs9906590 are close to known mutations that result in the loss of the enzymatic activity [40].

In all, the results suggest that several SNPs within HS3ST3A1 and HS3ST3B1, the genes encoding 3-OST$3 \mathrm{~A} 1$ and 3-OST-3B1, may cause variations in either gene expression levels or the enzymatic activity, and that this may result in variations in parasitaemia. Two mechanisms may explain how genetic variations in HSSST3A1 and HS3ST3B1 may result in variations in parasitaemia. Genetic variations in HSSST3A1 and HS 3ST3B1 that alter the 3-O sulphation of HS may affect i) the binding of $P$. falciparum antigen on host cells and/or ii) the pro-inflammatory response.

Together with previous reports [2-4,41], the results suggest that variations in the $3-\mathrm{O}$ sulphation catalyzed by 3-OST-3A1 and 3-OST-3B1 may affect both the binding of $P$. falciparum antigen on host cells and the parasite invasion rate. Highly sulphated HS has been shown to promote a productive invasion of cells by $P$. berghei sporozoites, whereas sporozoites migrate through cells harbouring low sulphated HS [2]. Since the 3-O sulphation is the last step of HS synthesis and occurs after the 2-O and 6-0 sulphation steps, one might assume that highly sulphated HS involved in the sporozoite invasion is 3-O sulphated. This hypothesis is consistent with the data showing that 2-O, 3-O, and $\mathrm{N}$ sulphate moieties participate in sporozoite CSP binding [41]. Although the $\mathrm{N}$-sulphation has been shown to be involved in rosette disruption [4-6], and although the Osulphation has not been reported to influence either the binding of $P$. falciparum antigen on the human erythrocyte surface or the merozoite invasion rate [3], the results showing the inhibition of merozoite invasion by heparin and other highly sulphated glycoconjugates $[42,43]$, and those showing the influence of the O-sulphation on hepatocyte invasion by sporozoites makes this hypothesis relevant.

Genetic variations in HSSST3A1 and HS3ST3B1 might also alter the immune response, and more specifically, the inflammatory response. Indeed, pro-inflammatory cytokine and chemokine binding to HS that depends on the sulphation profile of HS controls both the tissue targeting and the local accumulation of cytokines and chemokines [44,45]. Interestingly, CCR5 (chemokine (C-C motif) receptor 5 ) up-regulation was associated with the up-regulation of HSBST3A1 and HSSST3B1 in humans infected by HIV; the authors suggested either that interaction between HS and CCR5 causes up-regulation, or that the promoters of CCR5, HS3ST3A1 and HS3ST3B1 share a cis-regulatory motif binding the same transcription factor [46]. Since CCR5 and HS3ST3A1 have been associated with HIV infection [12,47], this suggests an interaction at the genetic level affecting resistance to HIV infection. By extension, this suggests that the interaction between immune genes and genes involved in HS biosynthesis may contribute to resistance against other infectious diseases, such as malaria. Additional investigations are 
needed to evaluate the role of such genetic interactions, and to elucidate how the sulphation profile of HS determines cytokine and chemokine binding.

\section{Conclusions}

This study shows that HS3ST3A1 and HS3ST3B1 are linked to $P$. falciparum parasitaemia, that rs 28470223 within the promoter of HS3ST3A1 is associated with $P$. falciparum parasitaemia, and that interactions between HS3ST3A1 and HS3ST3B1 polymorphisms alter P. falciparum parasitaemia. The results also indicate that rs28470223 and four mis-sense mutations within HS3ST3B1 strongly contribute to genetic interactions. This study also suggests that other genes involved in HS biosynthesis may affect malaria resistance. In this way, NDST1 that plays a major role in HS biosynthesis is located within the chromosome $5 \mathrm{q} 31-\mathrm{q} 33$, which is linked to P. falciparum parasitaemia [15].

\section{Additional material}

Additional file 1: Primer pairs and annealing temperatures used to amplify HS3ST3A1 and HS3ST3B 1

Additional file 2: The best multi-locus models identified with PGMDR. All the $P$ values were significant after applying a FDR of $5 \%$.

\begin{abstract}
Abbreviations
CCR5: Chemokine (C-C motif) receptor 5; CSP: Circumsporozoite protein; GRCh37: Genome Reference Consortium Human genome build 37; HIV: Human immunodeficiency virus; HS: Heparan sulphate; HSV-1: Herpes simplex virus 1; HSPG: Heparan sulphate proteoglycan; LD: Linkage Disequilibrium; MAF: Minor Allele Frequency; iRBC: infected red blood cells; PGMDR: Pedigree-based generalized multifactor dimensionality reduction
\end{abstract}

\section{Acknowledgements}

We thank all volunteer families of Bobo Dioulasso. We also thank Charles Chapple for critical reading of the manuscript. Assistance from the IGS-CNRS UPR2589 laboratory was greatly appreciated for DNA sequencing, and we particularly thank Jean-Michel Claverie, Chantal Abergel, Sabine Chenivesse, and Stephane Audic for their help.

\section{Author details}

'UMR928-TAGC, INSERM, 163 Av de Luminy, Marseille F-13288, France. ' $U M R-$ MD3, 27 Boulevard Jean Moulin, Marseille F-13385, France. ${ }^{3}$ Aix-Marseille University, Marseille F-13288, France.

\section{Authors' contributions}

AA carried out the molecular genetic studies, participated in the sequence alignment and performed the statistical analysis. SG participated in the molecular genetic studies and sequence alignment. SA participated in the statistical analysis. FF participated in the design of the study, and revised the results and the manuscript. PR performed the design of the study, supervised the experiments and the statistical analyses, and wrote the manuscript. All authors read and approved the final manuscript.

\section{Competing interests}

The authors declare that they have no competing interests.

Received: 30 January 2012 Accepted: 4 April 2012

Published: 4 April 2012
References

1. Sinnis P, Coppi A, Toida T, Toyoda H, Kinoshita-Toyoda A, Xie J, Kemp MM Linhardt RJ: Mosquito heparan sulphate and its potential role in malaria infection and transmission. J Biol Chem 2007, 282:25376-25384.

2. Coppi A, Tewari R, Bishop JR, Bennett BL, Lawrence R, Esko JD, Billker O, Sinnis P: Heparan sulphate proteoglycans provide a signal to Plasmodium sporozoites to stop migrating and productively invade host cells. Cell Host Microbe 2007, 2:316-327.

3. Kobayashi K, Kato K, Sugi T, Takemae H, Pandey K, Gong H, Tohya Y, Akashi H: Plasmodium falciparum BAEBL binds to heparan sulphate proteoglycans on the human erythrocyte surface. J Biol Chem 2010, 285:1716-1725.

4. Vogt AM, Winter G, Wahlgren M, Spillmann D: Heparan sulphate identified on human erythrocytes: a Plasmodium falciparum receptor. Biochem J 2004, 381:593-597.

5. Chen Q, Barragan A, Fernandez V, Sundstrom A, Schlichtherle M, Sahlen A, Carlson J, Datta S, Wahlgren M: Identification of Plasmodium falciparum erythrocyte membrane protein 1 (PfEMP1) as the rosetting ligand of the malaria parasite P. falciparum. J Exp Med 1998, 187:15-23.

6. Vogt AM, Barragan A, Chen Q, Kironde F, Spillmann D, Wahlgren M: Heparan sulphate on endothelial cells mediates the binding of Plasmodium falciparum-infected erythrocytes via the DBL1alpha domain of PfEMP1. Blood 2003, 101:2405-2411.

7. Prydz K, Dalen KT: Synthesis and sorting of proteoglycans. J Cell Sci 2000, 113(Pt 2):193-205.

8. Shukla D, Liu J, Blaiklock P, Shworak NW, Bai X, Esko JD, Cohen GH, Eisenberg RJ, Rosenberg RD, Spear PG: A novel role for 3-O-sulphated heparan sulphate in herpes simplex virus 1 entry. Cell 1999, 99:13-22.

9. Xu D, Tiwari V, Xia G, Clement C, Shukla D, Liu J: Characterization of heparan sulphate 3-O-sulphotransferase isoform 6 and its role in assisting the entry of herpes simplex virus type 1. Biochem J 2005, 385:451-459.

10. Shworak NW, Liu J, Petros LM, Zhang L, Kobayashi M, Copeland NG, Jenkins NA, Rosenberg RD: Multiple isoforms of heparan sulphate Dglucosaminyl 3-O-sulfotransferase. Isolation, characterization, and expression of human cdnas and identification of distinct genomic loci. $J$ Biol Chem 1999, 274:5170-5184.

11. Liu J, Shworak NW, Sinay P, Schwartz JJ, Zhang L, Fritze LM, Rosenberg RD: Expression of heparan sulphate D-glucosaminyl 3-O-sulfotransferase isoforms reveals novel substrate specificities. J Biol Chem 1999, 274:5185-5192.

12. Joubert BR, Lange EM, Franceschini N, Mwapasa V, North KE, Meshnick SR: A whole genome association study of mother-to-child transmission of HIV in Malawi. Genome Med 2010, 2:17.

13. Bobardt MD, Salmon P, Wang L, Esko JD, Gabuzda D, Fiala M, Trono D, Van der Schueren B, David G, Gallay PA: Contribution of proteoglycans to human immunodeficiency virus type 1 brain invasion. J Virol 2004, 78:6567-6584.

14. Aucan C, Traore Y, Tall F, Nacro B, Traore-Leroux T, Fumoux F, Rihet P: High immunoglobulin $\mathrm{G} 2$ ( $\lg \mathrm{G} 2$ ) and low lgG4 levels are associated with human resistance to Plasmodium falciparum malaria. Infect Immun 2000, 68:1252-1258.

15. Rihet P, Traore Y, Abel L, Aucan C, Traore-Leroux T, Fumoux F: Malaria in humans: Plasmodium falciparum blood infection levels are linked to chromosome 5q31-q33. Am J Hum Genet 1998, 63:498-505.

16. Kwiatkowski DP: How malaria has affected the human genome and what human genetics can teach us about malaria. Am J Hum Genet 2005, 77:171-192.

17. Flori L, Sawadogo S, Esnault C, Delahaye NF, Fumoux F, Rihet P: Linkage of mild malaria to the major histocompatibility complex in families living in Burkina Faso. Hum Mol Genet 2003, 12:375-378.

18. Sakuntabhai A, Ndiaye R, Casademont I, Peerapittayamongkol C, Rogier C, Tortevoye P, Tall A, Paul R, Turbpaiboon C, Phimpraphi W, Trape JF, Spiegel A, Heath S, Mercereau-Puijalon O, Dieye A, Julier C: Genetic determination and linkage mapping of Plasmodium falciparum malaria related traits in Senegal. PLoS One 2008, 3:e2000.

19. Timmann C, Evans JA, Konig IR, Kleensang A, Ruschendorf F, Lenzen J, Sievertsen J, Becker C, Enuameh Y, Kwakye KO, Opoku E, Browne EN, Ziegler A, Nurnberg P, Horstmann RD: Genome-wide linkage analysis of malaria infection intensity and mild disease. PLoS Genet 2007, 3:e48. 
20. Flori L, Kumulungui B, Aucan C, Esnault C, Traore AS, Fumoux F, Rihet P: Linkage and association between Plasmodium falciparum blood infection levels and chromosome 5q31-q33. Genes Immun 2003, 4:265-268.

21. Jepson A, Sisay-Joof F, Banya W, Hassan-King M, Frodsham A, Bennett S, Hill AV, Whittle H: Genetic linkage of mild malaria to the major histocompatibility complex in Gambian children: study of affected sibling pairs. BMJ 1997, 315:96-97.

22. Bongfen SE, Laroque A, Berghout J, Gros P: Genetic and genomic analyses of host-pathogen interactions in malaria. Trends Parasitol 2009, 25:417-422.

23. Verra F, Mangano VD, Modiano D: Genetics of susceptibility to Plasmodium falciparum: from classical malaria resistance genes towards genome-wide association studies. Parasite Immunol 2009, 31:234-253.

24. Hernandez-Valladares M, Naessens J, Iraqi FA: Genetic resistance to malaria in mouse models. Trends Parasitol 2005, 21:352-355.

25. Stevenson MM, Gros P, Olivier M, Fortin A, Serghides L: Cerebral malaria: human versus mouse studies. Trends Parasitol 2010, 26:274-275.

26. Hernandez-Valladares M, Naessens J, Gibson JP, Musoke AJ, Nagda S, Rihet $\mathrm{P}$, Ole-MoiYoi OK, Iraqi FA: Confirmation and dissection of QTL controlling resistance to malaria in mice. Mamm Genome 2004, 15:390-398.

27. Hernandez-Valladares $M$, Rihet $P$, ole-MoiYoi OK, Iraqi FA: Mapping of a new quantitative trait locus for resistance to malaria in mice by a comparative mapping approach with human chromosome 5q31-q33. Immunogenetics 2004, 56:115-117.

28. Lou XY, Chen GB, Yan L, Ma JZ, Mangold JE, Zhu J, Elston RC, Li MD: A combinatorial approach to detecting gene-gene and gene-environment interactions in family studies. Am J Hum Genet 2008, 83:457-467.

29. Rihet $P$, Abel L, Traore $Y$, Traore-Leroux T, Aucan C, Fumoux F: Human malaria: segregation analysis of blood infection levels in a suburban area and a rural area in Burkina Faso. Genet Epidemiol 1998, 15:435-450.

30. Rozen S, Skaletsky H: Primer3 on the WWW for general users and for biologist programmers. Methods Mol Biol 2000, 132:365-386.

31. Abecasis GR, Cherny SS, Cookson WO, Cardon LR: Merlin-rapid analysis of dense genetic maps using sparse gene flow trees. Nat Genet 2002, 30:97-101.

32. Lake SL, Blacker D, Laird NM: Family-based tests of association in the presence of linkage. Am J Hum Genet 2000, 67:1515-1525.

33. Abecasis GR, Cookson WO: GOLD-graphical overview of linkage disequilibrium. Bioinformatics 2000, 16:182-183.

34. Barrett JC, Fry B, Maller J, Daly MJ: Haploview: analysis and visualization of LD and haplotype maps. Bioinformatics 2005, 21:263-265.

35. Sham PC, Purcell S, Cherny SS, Abecasis GR: Powerful regression-based quantitative-trait linkage analysis of general pedigrees. Am J Hum Genet 2002, 71:238-253.

36. Abecasis GR, Cardon LR, Cookson WO: A general test of association for quantitative traits in nuclear families. Am J Hum Genet 2000, 66:279-292.

37. Ritchie MD, Hahn LW, Roodi N, Bailey LR, Dupont WD, Parl FF, Moore JH: Multifactor-dimensionality reduction reveals high-order interactions among estrogen-metabolism genes in sporadic breast cancer. Am J Hum Genet 2001, 69:138-147.

38. Benjamini Y, Yekutieli D: Quantitative trait Loci analysis using the false discovery rate. Genetics 2005, 171:783-790.

39. Jallow M, Teo YY, Small KS, Rockett KA, Deloukas P, Clark TG, Kivinen K, Bojang KA, Conway DJ, Pinder M, Sirugo G, Sisay-Joof F, Usen S, Auburn S, Bumpstead SJ, Campino S, Coffey A, Dunham A, Fry AE, Green A, Gwilliam R, Hunt SE, Inouye M, Jeffreys AE, Mendy A, Palotie A, Potter S, Ragoussis J, Rogers J, Rowlands K, Somaskantharajah E, Whittaker P, Widden C, Donnelly P, Howie B, Marchini J, Morris A, SanJoaquin M, Achidi EA, Agbenyega T, Allen A, Amodu O, Corran P, Djimde A, Dolo A, Doumbo OK, Drakeley C, Dunstan S, Evans J, Farrar J, Fernando D, Hien TT, Horstmann RD, Ibrahim M, Karunaweera N, Kokwaro G, Koram KA, Lemnge M, Makani J, Marsh K, Michon P, Modiano D, Molyneux ME, Mueller I, Parker M, Peshu N, Plowe CV, Puijalon O, Reeder J, Reyburn H, Riley EM, Sakuntabhai A, Singhasivanon P, Sirima S, Tall A, Taylor TE, Thera M, Troye-Blomberg M, Williams TN, Wilson M, Kwiatkowski DP. Genome-wide and fine-resolution association analysis of malaria in West Africa. Nat Genet 2009, 41:657-665.

40. Moon AF, Edavettal SC, Krahn JM, Munoz EM, Negishi M, Linhardt RJ, Liu J, Pedersen LC: Structural analysis of the sulfotransferase (3-o- sulfotransferase isoform 3) involved in the biosynthesis of an entry receptor for herpes simplex virus 1. J Biol Chem 2004, 279:45185-45193.

41. Pinzon-Ortiz C, Friedman J, Esko J, Sinnis P: The binding of the circumsporozoite protein to cell surface heparan sulphate proteoglycans is required for Plasmodium sporozoite attachment to target cells. J Biol Chem 2001, 276:26784-26791.

42. Kulane A, Ekre HP, Perlmann P, Rombo L, Wahlgren M, Wahlin B: Effect of different fractions of heparin on Plasmodium falciparum merozoite invasion of red blood cells in vitro. AmJTrop Med Hyg 1992, 46:589-594.

43. Xiao L, Yang C, Patterson PS, Udhayakumar V, Lal AA: Sulphated polyanions inhibit invasion of erythrocytes by plasmodial merozoites and cytoadherence of endothelial cells to parasitized erythrocytes. Infect Immun 1996, 64:1373-1378.

44. Lortat-Jacob H: Interferon and heparan sulphate. Biochem Soc Trans 2006, 34:461-464.

45. Lortat-Jacob $\mathrm{H}$ : The molecular basis and functional implications of chemokine interactions with heparan sulphate. Curr Opin Struct Biol 2009, 19:543-548.

46. Joubert BR, Franceschini N, Mwapasa V, North KE, Meshnick SR: Regulation of CCR5 expression in human placenta: insights from a study of motherto-child transmission of HIV in Malawi. PLoS One 2010, 5:e9212.

47. Kaur G, Mehra N: Genetic determinants of HIV-1 infection and progression to AIDS: susceptibility to HIV infection. Tissue Antigens 2009, 73:289-301.

\section{doi:10.1186/1475-2875-11-108}

Cite this article as: Atkinson et al:: Genetic variations in genes involved in heparan sulphate biosynthesis are associated with Plasmodium falciparum parasitaemia: a familial study in Burkina Faso. Malaria Journal 2012 11:108.

\section{Submit your next manuscript to BioMed Central and take full advantage of:}

- Convenient online submission

- Thorough peer review

- No space constraints or color figure charges

- Immediate publication on acceptance

- Inclusion in PubMed, CAS, Scopus and Google Scholar

- Research which is freely available for redistribution

Submit your manuscript a www.biomedcentral.com/submit
C) Biomed Central 